\title{
Study on the precision of the multiaperture scintillation sensor turbulence profiler (MASS) employed in the site testing campaign for the Thirty Meter Telescope
}

\author{
S. G. Els, ${ }^{1,2, \star}$ M. Schöck, ${ }^{2}$ J. Seguel, ${ }^{1}$ A. Tokovinin, ${ }^{1}$ V. Kornilov, ${ }^{3}$ R. Riddle, ${ }^{2}$ \\ W. Skidmore, ${ }^{2}$ T. Travouillon, ${ }^{2}$ K. Vogiatzis, ${ }^{2}$ R. Blum, ${ }^{4}$ E. Bustos, ${ }^{1}$ B. Gregory, ${ }^{1}$ \\ J. Vasquez, ${ }^{1}$ D. Walker, ${ }^{1}$ and P. Gillett ${ }^{2}$ \\ ${ }^{1}$ Cerro Tololo Inter-American Observatory, Casilla 603, La Serena, Chile \\ ${ }^{2}$ Thirty Meter Telescope Project, 2632 E. Washington Boulevard, Pasadena, California 91107, USA \\ ${ }^{3}$ Sternberg Astronomical Institute, Universitetsky prospekt 13, 119992 Moscow, Russia \\ ${ }^{4}$ National Optical Astronomy Observatory, 950 N. Cherry Avenue, Tucson, Arizona 85719, USA \\ ${ }^{*}$ Corresponding author: sels@ctio.noao.edu
}

Received 16 January 2008; revised 5 March 2008; accepted 7 March 2008; posted 20 March 2008 (Doc. ID 91713); published 2 May 2008

\begin{abstract}
The multiaperture scintillation sensor (MASS) has become a device widely employed to measure the altitude distribution of atmospheric turbulence. An empirical study is reported that investigates the dependence of the MASS results on the knowledge of the instrumental parameters. Also, the results of a side-by-side comparison of two MASS instruments are presented, indicating that MASS instruments permit measurements of the integrated seeing to a precision better than 0.05 arc sec and of the individual turbulence layer strength $C_{n}^{2}(h) d h$ to better than $10^{-14} \mathrm{~m}^{1 / 3}$. (C) 2008 Optical Society of America

OCIS codes: $\quad 010.0010,120.0120,280.0280,000.2170,010.1330$.
\end{abstract}

\section{Introduction}

Knowing the altitude distribution of optical turbulence in the earth's atmosphere has become a vital part in modern observational astronomy techniques. For the development and planning of astronomical instrumentation in general, and in particular of adaptive optics, as well as supporting operations of the existing observing facilities, devices measuring the vertical turbulence distribution-the turbulence profiles-are needed. Several instruments have been developed to measure the turbulence profiles, for example SCIDAR [1,2], SLODAR [3], or the multiaperture scintillation sensor (MASS) [4, 5$]$.

The last-MASS-measures the spatial light distribution of the flying shadows created by the scintillation of the incident light from a star on the turbulent atmospheric layers. From this, MASS com-

0003-6935/08/142610-09\$15.00/0

(C) 2008 Optical Society of America putes the differential scintillation indices among circular apertures of different sizes, which then allows the reconstruction of the turbulence profile [5].

MASS has become a standard tool to measure lowresolution turbulence profiles in current site testing and site qualification programs. However, even though the MASS instrument is now widely used, few studies exist on its instrumental precision $[6,7]$. The work in [8] demonstrates that the turbulence profiles obtained by MASS can be used to successfully predict the anisoplanatism of point spread functions in adaptive optics and indicates a good absolute accuracy. Here we present an empirical study of the MASS's precision, meaning the comparability between results obtained with different MASS units.

\section{Description of the Thirty Meter Telescope MASS-DIMM devices}

The site testing program for the Thirty Meter Telescope (TMT) has installed identical sets of equipment 
on five remote mountains to measure the atmospheric conditions affecting astronomical observations. The heart of each site testing system is a Cassegrain telescope with an aperture of $35 \mathrm{~cm}$, custom made by Teleskoptechnik Halfmann. The telescopes are mounted on towers, at an elevation of approximately $7 \mathrm{~m}$ above ground. Each telescope is equipped with a combined MASS and DIMM (dual in-line memory module) unit.

These units combine a MASS and a DIMM into a single instrument called MASS-DIMM (MD). Each MD is given a unit number (e.g., MD 1) to track its associated data archive, since occasional failures mean that a new unit needs to be deployed at a given site. Several mirrors are placed in the plane of the telescope's exit pupil to distribute the light into the MASS and the DIMM channels. Here we describe only the MASS; a detailed investigation of the DIMM channel is given elsewhere [9]. The segmentator for the MASS consists of one circular mirror, surrounded by three concentric reflective and tilted rings. These mirrors correspond to four apertures (A, B, C, D) with radii in the telescope pupil of $r_{A} \approx 2 \mathrm{~cm}, r_{B . \text { outer }} \approx$ $3 \mathrm{~cm}, \quad r_{C, \text { outer }} \approx 3 \mathrm{~cm}, \quad r_{D \text {,outer }} \approx 8 \mathrm{~cm}$. Each mirror directs the incoming light via another mirror into the corresponding detector. Photomultiplier tubes (PMTs) of type Hamamatsu R7400P are used as detectors. A more detailed description of the MD units can be found in [10].

The advantage of the combined MD device lie in its capability to measure the seeing from the ground to the top of the atmosphere in the DIMM channel and the turbulence profile from $500 \mathrm{~m}$ upward simultaneously, through the same atmosphere, since both the MASS and the DIMM channel observe the same star. The altitude elements of the MASS turbulence profile $C_{n}^{2}(h) d h$, or layers, are centered at $h=0.5,1,2,4,8,16 \mathrm{~km}$. The response of each MASS altitude layer goes approximately down to zero at the altitude of the closest adjacent layers, but there is a small overlap between layers. From the turbulence profile, the seeing from $500 \mathrm{~m}$ above ground and the isoplanatic angle $\left(\theta_{0}\right)$ are computed. Using simultaneously taken DIMM data then also permits us to compute the seeing within the lowest $500 \mathrm{~m}$ above the telescope.

This, in combination with the instrument's compactness, its usability on small aperture telescopes, and its lack of moving parts during operations make the MD a well-suited and robust tool for site characterization and site testing work.

\section{Sensitivity of MASS Results to Instrumental Parameters}

In its original version, the MD devices built at Cerro Tololo Inter-American Observatory (CTIO) were delivered with the real-time control and analysis software turbina. The turbina package did not allow the reprocessing of the collected data, and the accuracy of the results relied on proper knowledge of the instrumental parameters prior to observations.
These parameters are the instrument magnification, which is required for obtaining the projected size of the MASS apertures in the telescope entrance pupil; the Poisson parameter of each PMT channel $\left(P_{A, B, C . D}\right)$, which is a quantitative measure of the deviation of the photocounting device from Poisson statistics and should be close to 1; and, finally, the nonlinearity parameter for each PMT $\left(n L_{A B, C D}\right)$, which gives the detector dead time. An in-depth discussion of these parameters is given in [5].

Since late 2005 the software module atmos [10] has been available within turbina. It was developed to reprocess MASS data by using different instrument parameters, in case their behavior changed over time. This enhanced software enables us to perform a case study on how strongly the MASS results depend on the precision of the individual instrumental parameters. This study supplements that of [10], where a discussion about the importance of the individual instrumental settings on an analytical basis, that is, their impact on the weighting functions, is given.

We approach this topic by means of a sensitivity study: reprocessing MASS data by using different variations of instrumental settings. From the differences between the results of each case and a reference case, one can assess the impact of each parameter. A substantial amount of observational data has to be used for such a study in order to cover a wide range of atmospheric conditions. We used MASS data that were collected during the month of April 2006 with the TMT site testing equipment on Cerro Armazones in Chile (this telescope is referred to as T2). In all there were a total of more than 11,000 individual MASS data records. Table 1 shows all combinations of the instrument parameters with which these data were reprocessed. In the early phase of this project it was thought that the instrument magnification was around 14 (we know now that it is rather 15), and therefore case $1 \mathrm{c}$ was chosen to be the reference case to which all cases are compared. The results, the differences of the medians and means and the rms scatter of the seeing differences between the cases and the reference case, are shown in Table 1 . The highest median difference for $C_{n}^{2}(h) d h$ of a single layer was found to be $2 \times 10^{-14} \mathrm{~m}^{1 / 3}$, which occurred in the $500 \mathrm{~m}$ layer during the $P_{A}$ tests.

As can be seen from Table 1, the parameters affecting the MASS seeing the most are the magnification and the Poisson parameter of aperture A, $P_{A}$. This is consistent with the results of [10]: the system magnification should be known to better than 5\%-10\%. From our experience this can easily be achieved by using the method described in [10], backprojecting the MASS apertures onto the primary mirror. A good knowledge of the Poisson parameters is also necessary, even though to a lesser extent than for the system magnification. The Poisson parameters are measured in the TMT site survey each time a star is acquired, typically three times per 
Table 1. Summary of the Sensitivity Study of the Dependence of MASS Seeing on Instrumental Parameters ${ }^{a}$

\begin{tabular}{|c|c|c|c|c|c|c|c|c|c|c|c|c|c|}
\hline Case & Mag & $P_{A}$ & $P_{B}$ & $P_{C}$ & $P_{D}$ & $n L_{A}$ & $n L_{B}$ & $n L_{C}$ & $n L_{D}$ & $\begin{array}{c}\Delta \text { Median } \\
(\operatorname{arcsec})\end{array}$ & $\begin{array}{c}\Delta \text { Mean } \\
(\operatorname{arcsec})\end{array}$ & Slope & $\begin{array}{l}\mathrm{rms} \\
(\operatorname{arc} \\
\mathrm{sec})\end{array}$ \\
\hline $1 \mathrm{a}$ & 12 & 1.0 & 1.0 & 1.0 & 1.0 & 12.0 & 12.0 & 12.0 & 12.0 & 0.06 & 0.07 & 0.905 & 0.04 \\
\hline $1 \mathrm{c}$ & 14 & 1.0 & 1.0 & 1.0 & 1.0 & 12.0 & 12.0 & 12.0 & 12.0 & 0.00 & 0.00 & 1.00 & 0.00 \\
\hline $1 d$ & 15 & 1.0 & 1.0 & 1.0 & 1.0 & 12.0 & 12.0 & 12.0 & 12.0 & -0.02 & 0.03 & 1.057 & 0.02 \\
\hline $1 \mathrm{e}$ & 16 & 1.0 & 1.0 & 1.0 & 1.0 & 12.0 & 12.0 & 12.0 & 12.0 & -0.05 & 0.03 & 1.117 & 0.02 \\
\hline $2 \mathrm{c}$ & 14 & 1.075 & 1.0 & 1.0 & 1.0 & 12.0 & 12.0 & 12.0 & 12.0 & -0.04 & -0.03 & 0.987 & 0.02 \\
\hline $2 d$ & 14 & 1.100 & 1.0 & 1.0 & 1.0 & 12.0 & 12.0 & 12.0 & 12.0 & -0.04 & -0.03 & 0.991 & 0.02 \\
\hline $3 a$ & 14 & 1.0 & 1.025 & 1.0 & 1.0 & 12.0 & 12.0 & 12.0 & 12.0 & -0.01 & -0.01 & 0.998 & 0.01 \\
\hline $3 b$ & 14 & 1.0 & 1.050 & 1.0 & 1.0 & 12.0 & 12.0 & 12.0 & 12.0 & -0.01 & -0.01 & 0.995 & 0.01 \\
\hline $3 \mathrm{c}$ & 14 & 1.0 & 1.075 & 1.0 & 1.0 & 12.0 & 12.0 & 12.0 & 12.0 & -0.02 & -0.01 & 0.992 & 0.01 \\
\hline $3 d$ & 14 & 1.0 & 1.100 & 1.0 & 1.0 & 12.0 & 12.0 & 12.0 & 12.0 & -0.02 & -0.02 & 0.989 & 0.01 \\
\hline $5 a$ & 14 & 1.0 & 1.0 & 1.0 & 1.025 & 12.0 & 12.0 & 12.0 & 12.0 & $<0.01$ & $<0.01$ & 1.001 & 0.01 \\
\hline $5 b$ & 14 & 1.0 & 1.0 & 1.0 & 1.050 & 12.0 & 12.0 & 12.0 & 12.0 & $<0.01$ & $<0.01$ & 1.001 & 0.01 \\
\hline $5 c$ & 14 & 1.0 & 1.0 & 1.0 & 1.075 & 12.0 & 12.0 & 12.0 & 12.0 & $<0.01$ & $<0.01$ & 1.000 & 0.01 \\
\hline $5 \mathrm{~d}$ & 14 & 1.0 & 1.0 & 1.0 & 1.100 & 12.0 & 12.0 & 12.0 & 12.0 & $<0.01$ & $<0.01$ & 1.000 & 0.01 \\
\hline $6 a$ & 14 & 1.0 & 1.0 & 1.0 & 1.0 & 10.0 & 12.0 & 12.0 & 12.0 & $<0.01$ & $<0.01$ & 1.000 & 0.01 \\
\hline $6 \mathrm{~b}$ & 14 & 1.0 & 1.0 & 1.0 & 1.0 & 11.0 & 12.0 & 12.0 & 12.0 & $<0.01$ & $<0.01$ & 1.000 & $<0.01$ \\
\hline $6 \mathrm{c}$ & 14 & 1.0 & 1.0 & 1.0 & 1.0 & 13.0 & 12.0 & 12.0 & 12.0 & $<0.01$ & $<0.01$ & 1.000 & $<0.01$ \\
\hline $6 \mathrm{~d}$ & 14 & 1.0 & 1.0 & 1.0 & 1.0 & 14.0 & 12.0 & 12.0 & 12.0 & $<0.01$ & $<0.01$ & 1.000 & $<0.01$ \\
\hline $7 \mathrm{a}$ & 14 & 1.0 & 1.0 & 1.0 & 1.0 & 12.0 & 10.0 & 12.0 & 12.0 & $<0.01$ & $<0.01$ & 1.000 & 0.01 \\
\hline $7 \mathrm{~b}$ & 14 & 1.0 & 1.0 & 1.0 & 1.0 & 12.0 & 11.0 & 12.0 & 12.0 & $<0.01$ & $<0.01$ & 1.000 & $<0.01$ \\
\hline $7 \mathrm{c}$ & 14 & 1.0 & 1.0 & 1.0 & 1.0 & 12.0 & 13.0 & 12.0 & 12.0 & $<0.01$ & $<0.01$ & 1.001 & $<0.01$ \\
\hline $7 d$ & 14 & 1.0 & 1.0 & 1.0 & 1.0 & 12.0 & 14.0 & 12.0 & 12.0 & $<0.01$ & $<0.01$ & 1.001 & $<0.01$ \\
\hline
\end{tabular}

${ }^{a}$ More than 11,000 individual MASS observations taken at Cerro Armazones during April 2006 by the TMT T2 site testing system were used for this study. "Mag" indicates the instrument magnification. $P$ and $n_{L}$ are the Poisson and nonlinearity parameters of the subaperture PMT. All cases were compared with the results obtained in case 1c. $\Delta$ median indicates the difference between the median of a case seeing and the median of the reference case seeing. $\Delta$ mean indicates the corresponding difference between the mean seeing of these cases. The slope was obtained by a linear fit to the seeing data, e.g., case 1a versus case 1c.

night. Figure 1 shows three years of measurements of the Poisson parameters of the PMTs used in the MD device, which is deployed on the TMT candidate site T1 on Cerro Tolar in northern Chile. During the first months, the integration times used for the PMT tests were only $1 \mathrm{~s}$, explaining their higher scatter. Later, this exposure time was changed to $10 \mathrm{~s}$, demonstrating that the scatter is dominated by photon statistics. It turns out that the PMTs show a very stable behavior of their Poisson parameters, making it possible to use the average value for each channel for the reprocessing of the MASS data.

We also investigated the impact of the spectral response function of the instrument and the telescope. Various spectral response functions are given in [11] and are reproduced in Fig. 2 . Their main differences occur toward the blue end of the spectrum. The spectral cutoff toward the red is dominated by the PMT response. The cutoff in the blue is due to the optical surfaces in the optical train, and not all MD units used in the TMT site testing employ the same optical parts. The segmentator optics in certain TMT MD units were made of bronze coated with aluminum; others were made of acrylic through which the light passes twice. Also, the mirrors redirecting the beam into the PMTs differ between certain units; they have either dielectric or aluminum coatings. Each of these components alters the overall spectral response of the MASS system. More information can be found in $[11,12]$.

We compared data obtained with various site testing systems, to cover the possible combinations of 


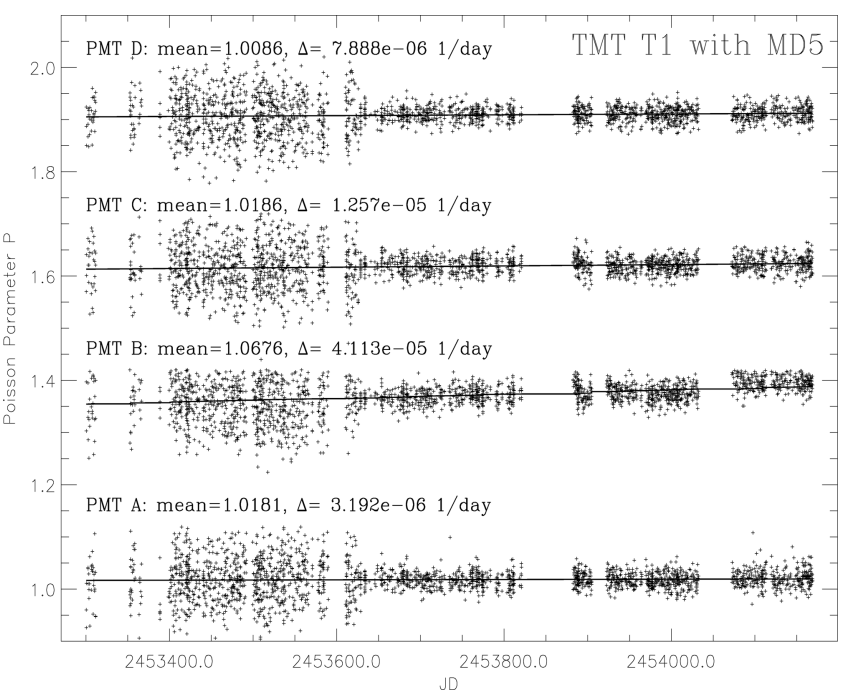

Fig. 1. Poisson parameter $P$ of the PMTs as measured by the detector tests obtained with MD 5, which was mounted on the T1 site testing telescope on Cerro Tolar. The plot shows data taken between 22 October 2007 and 9 March 2007, with more than 2290 samples. Data for the B, C, and D channels are offset from the A channel data by $0.3,0.6$, and 0.9 , respectively. The solid lines indicate linear fits to the data of each channel. The mean $\langle P\rangle$ values and the linear slopes $\Delta$ are shown above the respective channel data.

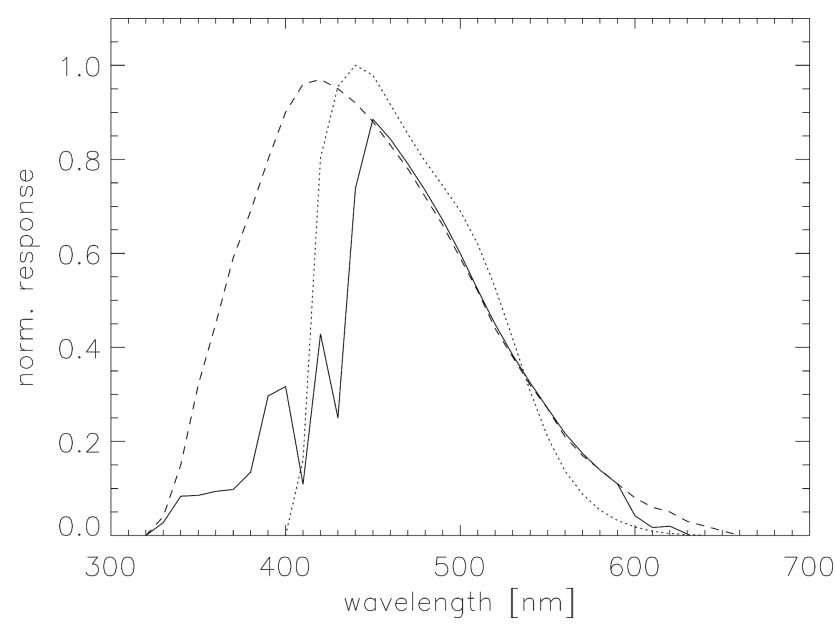

Fig. 2. Spectral response curves used for reprocessing MASS data, reproduced from [11]. The solid curve is referred to as without.crv, dotted as mass.crv, and dashed as eso_md.crv. instrument optics used within the TMT site testing effort. We used the data collected between July and September 2006 with MD 6 on Cerro Armazones (Chile), MD 10 on San Pedro Mártir (Mexico) and MD 8 at 13 North (Mauna Kea). These data were processed by using different spectral response functions and compared with the data processed by using the spectral response function mass. crv as the reference. The results are summarized in Table 2. We note that the highest change found for the median turbulence strength of the individual layers is $8 \times 10^{-15} \mathrm{~m}^{1 / 3}$, in the case of the MD 6 data at $2 \mathrm{~km}$. However, it can be concluded that even in the worst case, in which the data processed with a spectral response function ranging far into the blue are compared with the results obtained with a function having its cutoff more toward the red, does not introduce seeing biases larger than 0.02 arc sec and isoplanatic angle biases larger than 0.08 arc sec.

\section{Side-by-Side Comparison of Two MASS Devices}

Section 3 described to what level the instrumental parameters have to be known in order to obtain reliable results with the MASS instrument. In this section, we describe an experiment from which we deduce the final field precision of the MASS instruments used in the TMT site testing.

The TMT site testing project conducted a side-byside comparison campaign on Cerro Tololo between August and October of 2004. The DIMM data that were obtained during that campaign were presented in [9]. The experimetal setup consisted of two TMT site testing telescopes, T2 and T3, which were both installed $7 \mathrm{~m}$ above the ground and horizontally separated by approximately $5 \mathrm{~m}$ in an east-west direction, close to the northern edge of the Tololo summit area. With each telescope mounted on its own tower, this setup resembles well the setup on the TMT candidate sites.

The T2 telescope was equipped with MD 6, the T3 telescope with MD 2. During the campaign, both telescopes were pointing at the same stars. Data acquisition was not synchronized between $\mathrm{T} 2$ and $\mathrm{T} 3$, but as the robotic system triggers a new observation approximately every $70-90 \mathrm{~s}$, the time delay between a T2 and a T3 observation is generally less than $1 \mathrm{~min}$. The MASS devices operated in their generic

Table 2. Summary of the Sensitivity Study of the Dependence of MASS Seeing and Isoplanatic Angle $\theta_{0}$ on Spectral Response ${ }^{a}$

\begin{tabular}{|c|c|c|c|c|c|c|c|c|}
\hline Telescope & MASS unit & Spectral Response & $\begin{array}{c}\Delta \text { Median } \\
\text { Seeing }(\operatorname{arc~sec})\end{array}$ & $\begin{array}{c}\Delta \text { Mean } \\
\text { Seeing }(\operatorname{arc~sec})\end{array}$ & $\begin{array}{l}\mathrm{rms} \text { Seeing } \\
(\operatorname{arcsec})\end{array}$ & $\begin{array}{l}\Delta \text { Median } \theta_{0} \\
\quad(\operatorname{arcsec})\end{array}$ & $\begin{array}{l}\Delta \text { Mean } \theta_{0} \\
(\operatorname{arcsec})\end{array}$ & $\begin{array}{c}\operatorname{rms} \theta_{0} \\
(\operatorname{arc~sec})\end{array}$ \\
\hline $\mathrm{T} 2$ & MD 6 & without.crv & -0.02 & -0.02 & 0.10 & -0.01 & 0.02 & 0.67 \\
\hline \multirow[t]{2}{*}{$\mathrm{T} 4$} & MD 10 & without.crv & -0.01 & -0.01 & 0.07 & -0.05 & -0.04 & 0.21 \\
\hline & & eso_md.crv & $<0.01$ & $<0.01$ & 0.08 & 0.08 & 0.06 & 0.16 \\
\hline T6 & MD 8 & without.crv & -0.01 & -0.02 & 0.14 & -0.01 & -0.02 & 1.43 \\
\hline
\end{tabular}

${ }^{a}$ All numbers were obtained by comparison with the results based on the spectral response function mass. Crv. To obtain these results, data taken between July and September 2006 were used, resulting in more than 7200, 10,100 and 12,100 individual data records for T2, $\mathrm{T} 4$, and T6, respectively. 
mode; i.e., scintillation indices are computed from $1 \mathrm{~min}$ samples of $1 \mathrm{~ms}$ exposures [5]. Only data from T2 and T3 that were within $30 \mathrm{~s}$ of each other were selected for the study presented here, resulting in a total of 11,543 MASS data points.

No interpolation of these data was done to match the times of observations to obtain the results reported here. However, to check on the effects of timing offsets, we did two cross checks. First, we compared the results for the seeing precision from the data taken within $30 \mathrm{~s}$ with results from data that were taken within 5,10 , and $20 \mathrm{~s}$. The differences in the seeing differences were found to be less than $10 \%$. Second, we interpolated the T2 observations to the times of observations of T3. Also in that case, the agreement between these results was better than $10 \%$. We found in both tests that results computed from simultaneous data do not always improve the agreement between the two systems. In the first check we lower the sample size, which then affects the statistics, and in the second check interpolation errors are likely to be introduced.

In addition, the MASS units do not sample the same part of the atmosphere; the optical beams are not coincident. The MASS units have a field of view of $1.5 \times$ arc min, which corresponds to a horizontal extent of $22 \mathrm{~cm}$ at a distance of $500 \mathrm{~m}$ from the device and $4.4 \mathrm{~m}$ at a distance of $10 \mathrm{~km}$. The beams do not begin to significantly overlap closer than several kilometers from the telescopes. So even if both telescopes were to be perfectly synchronized in time, we would not expect the measurements to be identical.

Therefore, we expect the errors in the differences in seeing measured by two MASS devices, which are reported in the following, and which are based on the data taken within $30 \mathrm{~s}$ of each other, to be better than $10 \%$.

Data were reprocessed using the turbina/atmos package [11], employing the proper instrumental parameters of each MD unit during the time of observation.

Here, we first show results computed from data covering the time period between 17 September 2007 and 28 October 2004, consisting of 8777 data records. Before that date, some vignetting of the MASS apertures was present, which allows us to investigate its impact on MASS data in Subsection 4.D.

\section{A. Turbulence Profile $C_{n}^{2}(h) d h$}

The main output from the MASS are the turbulence integrals $C_{n}^{2}(h) d h$ in each layer. In the panels of Fig. 3, the scatter plots of the T2 and T3 MASS results for each layer are shown. The results are
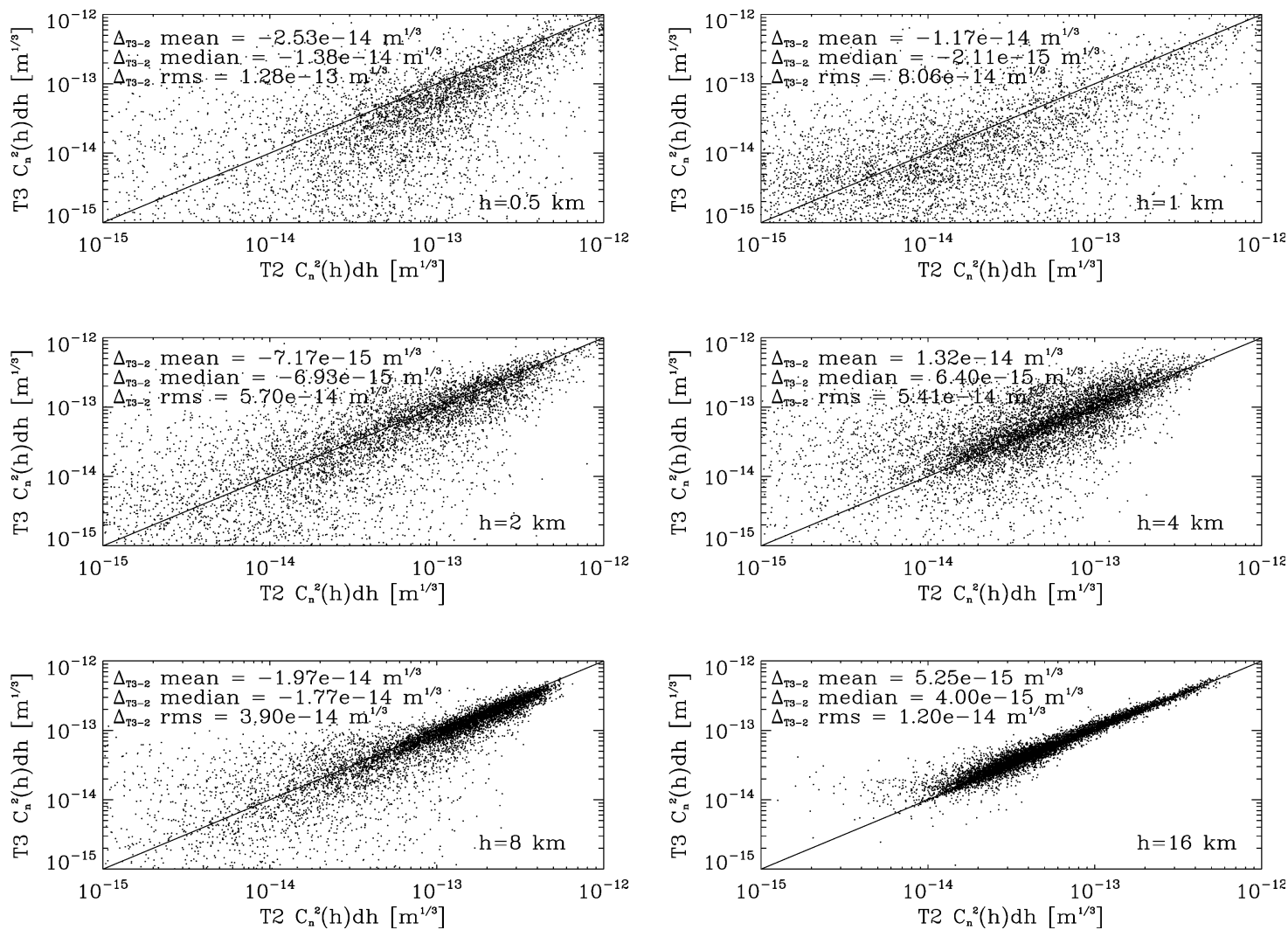

Fig. 3. Scatter plots of the $C_{n}^{2}(h) d h$ measurements for each layer, obtained by the T2 and T3 MDs during the Tololo 2004 campaign between 17 September and 28 October 2004. Only data were used that were taken at T2 and T3 within $30 \mathrm{~s}$, resulting in more than 8777 data records. The 1:1 correlation is marked by the solid line. 
Table 3. Main Results from the Side-by-Side Comparison of Two TMT Site Survey Instruments, T2 and T3, on Cerro Tololo ${ }^{a}$

\begin{tabular}{lccc}
\hline \multicolumn{1}{c}{ MASS Result } & $\left(\mathrm{T}_{3}-\mathrm{T}_{2}\right)_{\text {median }}$ & $\left(\mathrm{T}_{3}-\mathrm{T}_{2}\right)_{\text {mean }}$ & $\left(\mathrm{T}_{3}-\mathrm{T}_{2}\right)_{\mathrm{rms}}$ \\
\hline Seeing (arc sec) & -0.040 & -0.049 & 0.073 \\
$\theta_{0}(\operatorname{arcsec})$ & 0.001 & 0.003 & 0.096 \\
$\tau_{0} \times(\mathrm{ms})$ & 0.020 & $-2.53 \times 10^{-14}$ & 0.166 \\
$C_{n}^{2} d h, h=0.5 \mathrm{~km}\left(\mathrm{~m}^{1 / 3}\right)$ & $-1.37 \times 10^{-14}$ & $-1.17 \times 10^{-14}$ & $1.28 \times 10^{-13}$ \\
$C_{n}^{2} d h, h=1 \mathrm{~km}\left(\mathrm{~m}^{1 / 3}\right)$ & $-2.11 \times 10^{-15}$ & $-7.17 \times 10^{-15}$ & $8.06 \times 10^{-14}$ \\
$C_{n}^{2} d h, h=2 \mathrm{~km}\left(\mathrm{~m}^{1 / 3}\right)$ & $-6.93 \times 10^{-15}$ & $1.32 \times 10^{-15}$ & $5.70 \times 10^{-14}$ \\
$C_{n}^{2} d h, h=4 \mathrm{~km}\left(\mathrm{~m}^{1 / 3}\right)$ & $6.40 \times 10^{-15}$ & $-1.97 \times 10^{-15}$ & $5.41 \times 10^{-14}$ \\
$C_{n}^{2} d h, h=8 \mathrm{~km}\left(\mathrm{~m}^{1 / 3}\right)$ & $-1.77 \times 10^{-14}$ & $5.25 \times 10^{-15}$ & $3.90 \times 10^{-14}$ \\
$C_{n}^{2} d h, h=16 \mathrm{~km}\left(\mathrm{~m}^{1 / 3}\right)$ & $4.00 \times 10^{-15}$ & $1.20 \times 10^{-14}$ \\
\hline
\end{tabular}

${ }^{a}$ The $\tau_{0}$ results were obtained only from data for which $0<\tau_{0, \mathrm{~T} 2, \mathrm{~T} 3}<5 \mathrm{~ms}$.
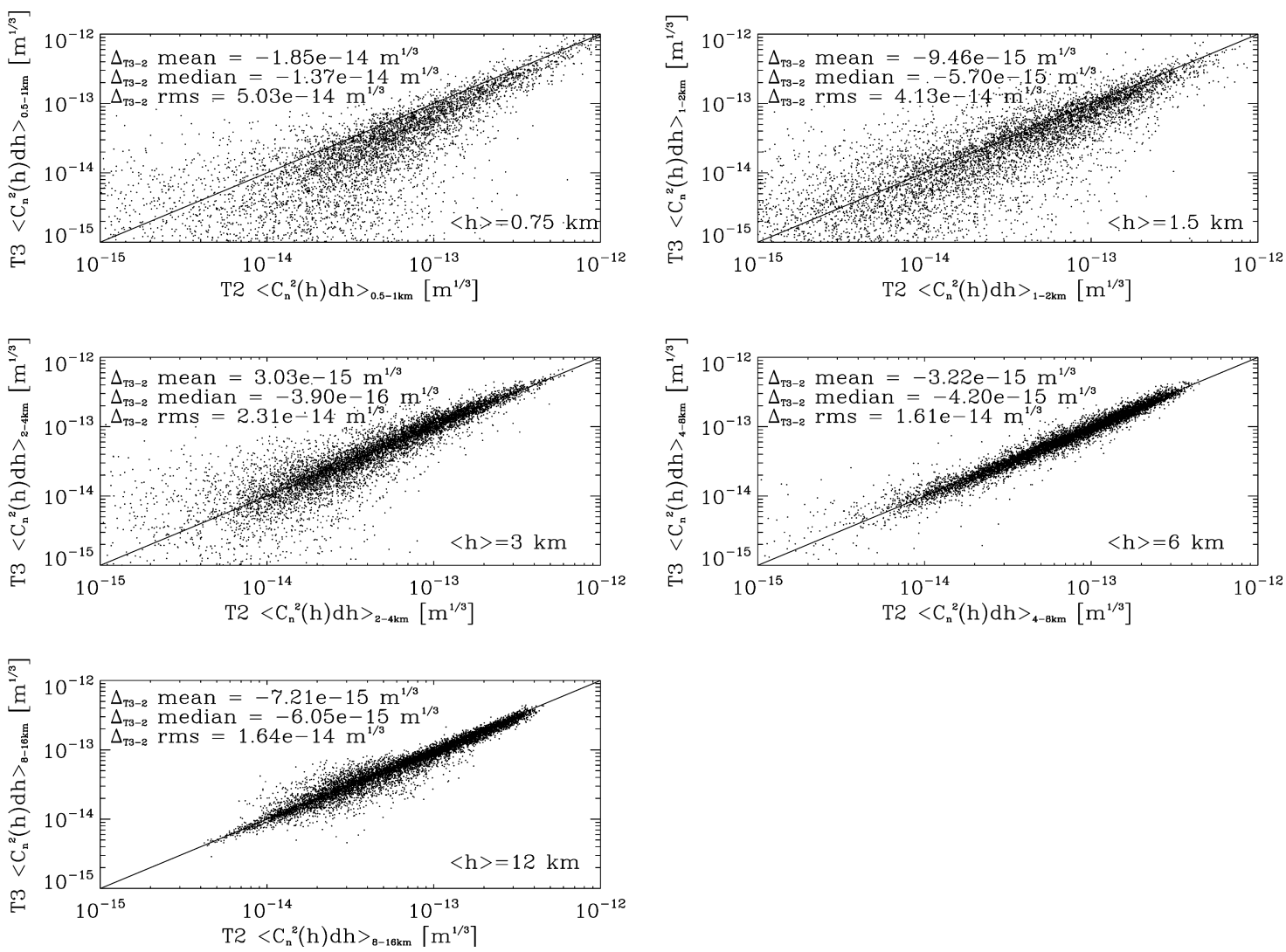

Fig. 4. Scatter plots like those in Fig. $\underline{3}$, but this time for the average of two adjacent layers.

summarized in Table 3. The rms scatter around the 1:1 correlation is also shown and can be assumed to be the precision of the individual measurement, assuming that both systems trace the same atmosphere, which is reasonable, as both instruments are only separated by a few meters.

It can be seen that the scatter for the lower layers is higher than for the upper layers. From about $h \geq$ $4 \mathrm{~km}$ onward, the correlations between the results of the two MASS devices is much more pronounced. However, it should be noted that the differences between the median values is always less than $1.4 \times 10^{-14} \mathrm{~m}^{1 / 3}$.

These differences of the turbulence strengths measured by the two MASS units is in part due to the fact that both instruments do trace slightly different atmospheric slabs and observations are not perfectly matched in time. But, also, as the MASS is solving an inverse problem on a six point altitude grid, it is possible that the solution for the turbulence can shift amplitude between adjacent layers. For example, assume some turbulence to be located at an altitude of $1.5 \mathrm{~km}$; then a small variation in the signal could cause one MASS to place a larger contribution of that turbulence at $2 \mathrm{~km}$, whereas the other MASS finds a solution that locates more turbulence at $1 \mathrm{~km}$. Both solutions would be valid within the noise of each of the instruments. To see whether this effect does account for the observed scatter, Fig. 4 shows the correlation of the averaged values $\left\langle\overline{C_{n}^{2}} d h(h)\right\rangle$ 
of adjacent layers, e.g., $\left\langle C_{n}^{2} d h(h)\right\rangle_{0.5-1 \mathrm{~km}}=0.5 \times$ $\left(C_{n}^{2}(h=0.5 \mathrm{~km}) d h+C_{n}^{2}(h=1 \mathrm{~km}) d h\right)$. As can be seen, the scatter decreases by up to factors of 4 . So indeed these jumping layers do account in part for the observed scatter. Some part of this improvement will be due to noise reduction by averaging. Note that this averaging of layers does not significantly change the median differences between the two MASS systems.

\section{B. MASS Seeing and Isoplanatic Angle}

The comparison of the seeing and the isoplanatic angle are shown in Fig. $\underline{5}$ and are also summarized in Table 3. As both values are integrated from the reconstructed turbulence profiles, they should agree with the previous results in the sense of any difference between the two telescopes. The agreement of seeing between the two MASS units is found to be 0.049 arc sec. The T2 MASS finds on average stronger turbulence values than T3 in most layers. This can also be seen in the seeing comparison. However, with an agreement of 0.001 arc sec, there is almost no difference between the isoplanatic angles because of the $h^{5 / 3}$ altitude weighting factor in the calculation of this parameter. The very low scatter of the isoplanatic angle results from the higher precision of the MASS results for the high-altitude layers.

\section{Coherence Time}

The coherence time $\tau_{0}$ is measured by the MASS as outlined by [4]. It assumes that the coherence time is proportional to a differential exposure scintillation index to the $(-0.6)$ power. A more recent study by [13] comes to the conclusion that these MASS-based $\tau_{0}$ estimates can be improved by the knowledge of the ground layer wind speed and are suspected to have an absolute accuracy of $20 \%$. Any $\tau_{0}$ estimation depends strongly on the knowledge of the proper vertical wind speed profile at the time of observation, which is generally unknown. This will be the topic of a future investigation.

The current study is therefore only investigating the relative agreement between two separate MASS devices operated under same conditions. The results shown here were generated by the atmos package, and no further adjustment was applied. These results therefore have to be taken with care and only indicate that two MASS units measure $\tau_{0}$ as defined by [4] to within a certain precision.

In Fig. 6 the correlation of $\tau_{0}$ as measured by T2 and T3 are shown, and results are tabulated in Table 3. The correlation between the two systems is best at values $\tau_{0} \lesssim 5 \mathrm{~ms}$ because of the weighting function of the differential exposure scintillation indices, which shows the proper $v^{5 / 3}$ increase only in the regime in which the aperture size is less than the Fresnel radius, as was shown in [4]. Assuming a wind speed of $v=20 \mathrm{~m} / \mathrm{s}$, the assumption that the Fresnel radius is smaller than $v \tau_{0}$ breaks down for our MASS devices with maximum aperture diameters of $8 \mathrm{~cm}$ at $\tau_{0} \gtrsim 4 \mathrm{~ms}$. The good agreement between this theoretical value and the limit found in Fig. $\underline{6}$, up to which the two systems correlate well, indicates that the method described in [4] to measure

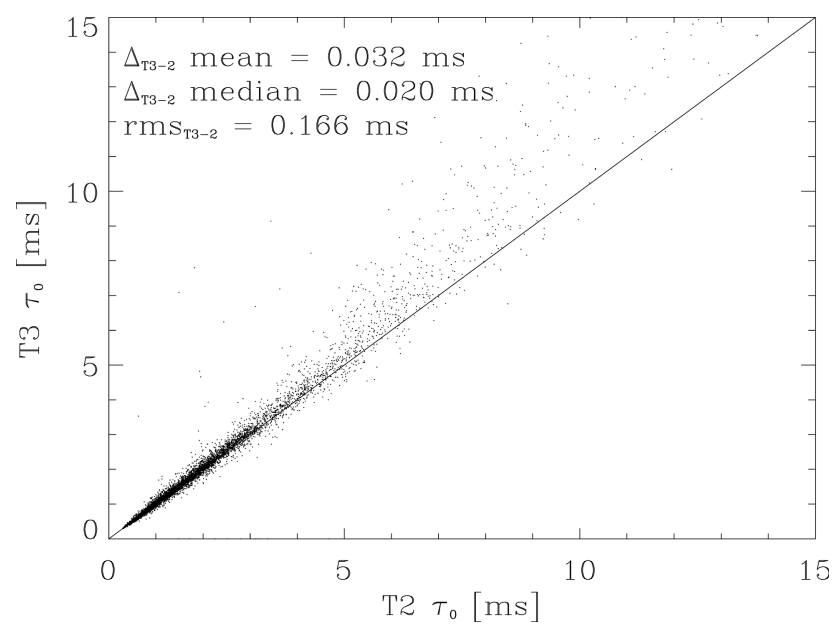

Fig. 6. Comparison of the coherence times $\left(\tau_{0}\right.$, as defined in [4]) measured by T2 and T3 during the Tololo 2004 campaign (see also Fig. 3). The values were computed only from data for which $0<\tau_{0, T 2, T 3}<5 \mathrm{~ms}$, corresponding to more than 8080 data records.
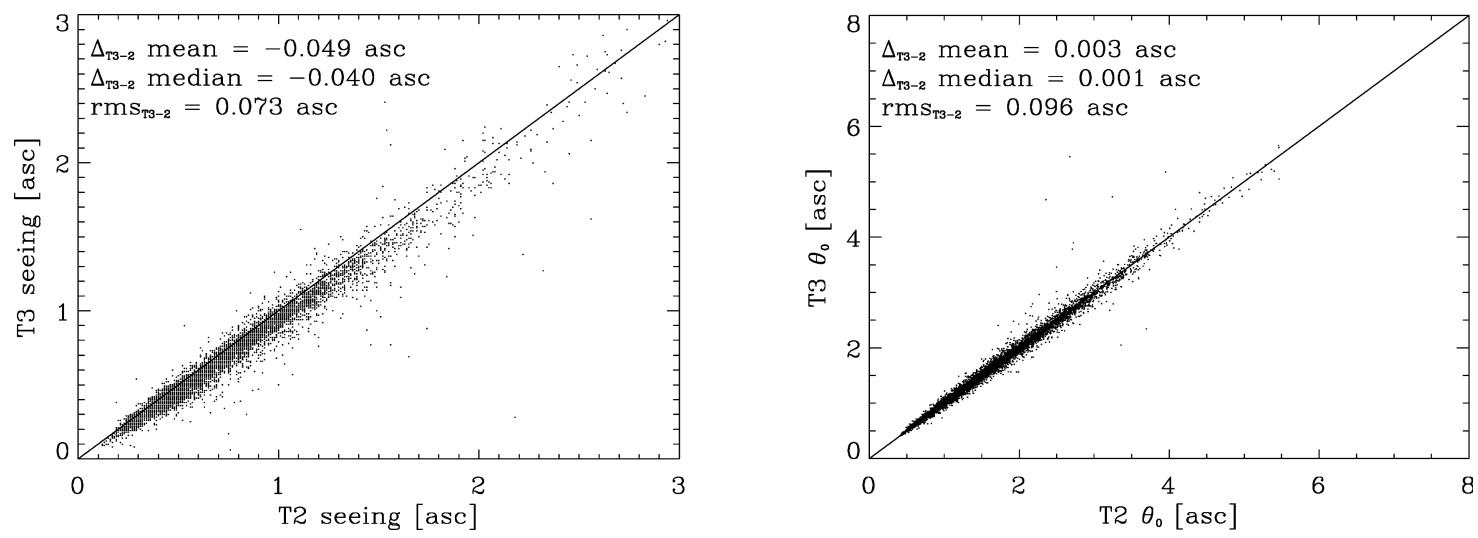

Fig. 5. Scatter plots of turbulence parameters measured by T2 and T3 during the Tololo 2004 campaign (see also Fig. 3 ). Left, MASS seeing; right, isoplanatic angle $\theta_{0}$. 
$\tau_{0}$ is indeed valid only up to about $5 \mathrm{~ms}$. The numbers shown in Fig. 6 and Table 3 were therefore computed only for data with $\tau_{0}$ less than $5 \mathrm{~ms}$.

\section{Vignetted MASS Data}

As the concept of MASS is based on accurate photometry, any vignetting of the optical train of the MASS instrument is highly undesirable. Therefore, care has to be taken during the setup and alignment of the instrument at the telescope. The MASS apertures have to be aligned onto the telescope pupil in a way that neither the edge of the pupil image of the primary mirror nor the secondary (with its support struts) are falling onto them. During the tests performed in the Tololo 2004 campaign, this alignment was found to be imperfect twice and resulted in a vignetting of MASS apertures.

Identifying periods of vignetting can be done by analyzing the time series of the flux ratios of the four MASS channels. In Fig. 7: the flux ratios $A / B, B / C$, and $C / D$ of the two MASSS systems are shown. A change of vignetting of a single aperture will show up as a discrete jump in one of these three time

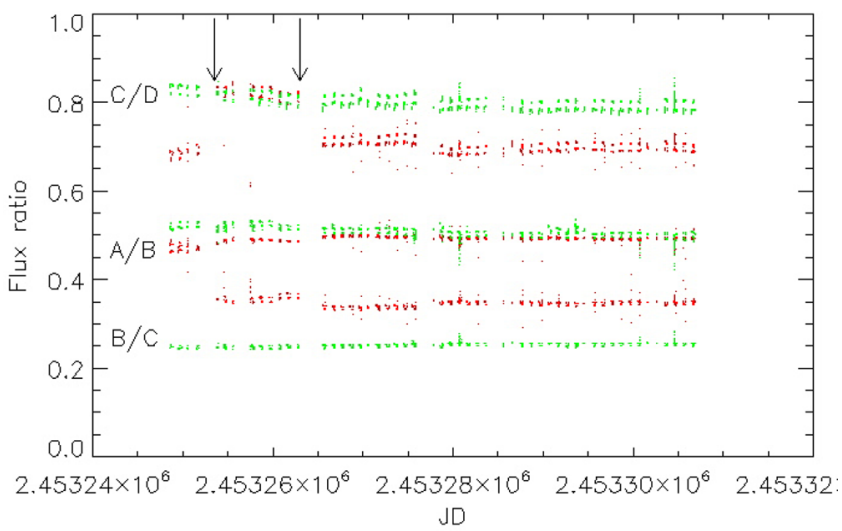

Fig. 7. (Color online) MASS aperture flux ratios from the T2 (red or darker gray) and T3 (green or lighter gray) MASS systems between 30 August and 28 October 2004, when both systems were mounted close to each other at CTIO. T2 shows a vignetting of the $\mathrm{D}$ aperture between 5 and 14 September 2004 (dates marked by the arrows) affecting 1826 data points. Its A aperture also seems to be vignetted during the four nights before that period. series. By looking at the $C / D$ ratio in Fig. 7 , one finds that the T2 (red online) data are displaced by about $10 \%-20 \%$ between the times marked by the arrows, corresponding to 5 and 14 September 2007. This displacement indicates that the $\mathrm{D}$ aperture was vignetted by about 10\%-20\% during this period of time. During the four nights before 5 September 2007 vignetting was present in the A aperture of T2. On both occasions the vignetting was caused by an imperfect alignment of the MASS apertures on the telescope entrance aperture, causing obstruction of the MASS apertures by telescope structures, i.e., the secondary support struts or the secondary mirror itself.

The MASS data obtained during these periods thus allow a direct comparison of the results taken with a vignetted and an unvignetted system. The comparison uses 2761 simultaneous data points taken during the period in which T2 showed some vignetting, that is, before 14 September 2004. We focus in the following only on the times during which a vignetting of the $D$ aperture was present, as a vignetting of the A aperture is much more difficult to introduce and is therefore very unlikely (nevertheless it can happen). Our results are summarized in Table 4. It turns out that the differences of the medians of the seeing and the isoplanatic angle values taken under vignetted and unvignetted conditions are almost identical. We conclude that a small vignetting of the $\mathrm{D}$ aperture, affecting the flux measurement by $\lesssim 10 \%-20 \%$, does not introduce any significant bias.

\section{Summary and Conclusion}

The results of an empirical investigation of the MASS precision have been presented. An analysis of the sensitivity of MASS results on the instrumental parameters shows that a good knowledge of these is necessary to obtain reliable turbulence results using the MASS. Our findings confirm the theoretical results of [10]. We find that modest differences in the MASS instrumental settings between those used in the analysis and those actually corresponding to the real MASS configuration introduce biases in the MASS seeing measurement of up to 0.06 arc sec. If the instrumental setup is carefully monitored and

Table 4. Site-by-Site Comparison Results for Data Taken between 5 and 14 September $2007^{a}$

\begin{tabular}{|c|c|c|c|c|}
\hline MASS result & $(\mathrm{T} 2-\mathrm{T} 3)_{\text {median, vignetted }}$ & $(\mathrm{T} 2-\mathrm{T} 3)_{\text {median, unvignetted }}$ & $\mathrm{rms}_{\text {vignetted }}$ & $\mathrm{rms}_{\text {unvignetted }}$ \\
\hline Seeing (arc sec) & 0.05 & 0.04 & 0.11 & 0.07 \\
\hline$\theta_{0}(\operatorname{arcsec})$ & 0.02 & $<0.01$ & 0.06 & 0.10 \\
\hline$C_{n}^{2} d h, h=0.5 \mathrm{~km}\left(\mathrm{~m}^{1 / 3}\right)$ & $4.51 \times 10^{-15}$ & $1.37 \times 10^{-14}$ & $2.02 \times 10^{-13}$ & $1.27 \times 10^{-13}$ \\
\hline$C_{n}^{2} d h, h=1 \mathrm{~km}\left(\mathrm{~m}^{1 / 3}\right)$ & $1.35 \times 10^{-15}$ & $2.11 \times 10^{-15}$ & $1.94 \times 10^{-13}$ & $8.06 \times 10^{-14}$ \\
\hline$C_{n}^{2} d h, h=2 \mathrm{~km}\left(\mathrm{~m}^{1 / 3}\right)$ & $1.80 \times 10^{-16}$ & $6.93 \times 10^{-15}$ & $1.12 \times 10^{-13}$ & $5.70 \times 10^{-14}$ \\
\hline$C_{n}^{2} d h, h=4 \mathrm{~km}\left(\mathrm{~m}^{1 / 3}\right)$ & $-1.50 \times 10^{-15}$ & $-6.40 \times 10^{-15}$ & $6.53 \times 10^{-14}$ & $5.41 \times 10^{-14}$ \\
\hline$C_{n}^{2} d h, h=8 \mathrm{~km}\left(\mathrm{~m}^{1 / 3}\right)$ & $2.97 \times 10^{-14}$ & $1.77 \times 10^{-14}$ & $7.05 \times 10^{-14}$ & $3.90 \times 10^{-14}$ \\
\hline$C_{n}^{2} d h, h=16 \mathrm{~km}\left(\mathrm{~m}^{1 / 3}\right)$ & $-6.60 \times 10^{-15}$ & $-4.00 \times 10^{-15}$ & $1.90 \times 10^{-14}$ & $1.20 \times 10^{-14}$ \\
\hline
\end{tabular}

${ }^{a}$ As in Table 3, 1826 data records, for which T2 showed some vignetting. These are compared with the simultaneously obtained data taken with T3, which was not vignetted. 
the data are processed using the correct values for all observations, this bias is an upper limit and the precision is expected to be much better.

A side-by-side comparison campaign of two MASS systems allowed us to assess the field precision of the MASS instrument, which is found to be better than 0.05 arc sec in seeing and better than $1.4 \times 10^{-14} \mathrm{~m}^{1 / 3}$ in $C_{n}(h)^{2} d h$. These results hold even when data were taken with a slight vignetting $(\sim 10 \%-20 \%$ of the surface of the outer aperture).

Thus the MASS gives a robust result that can be compared from site to site, and it is relatively insensitive to minor configuration discrepancies. We conclude that the MASS data collected by the site testing project for TMT is of high quality and can be used to compare the turbulence profile above the different sites.

We thank the Cerro Tololo Interamerican Observatory staff for their support and hospitality. The TMT Project gratefully acknowledges the support of the TMT partner institutions. They are the Association of Canadian Universities for Research in Astronomy (ACURA), the California Institute of Technology, and the University of California. This work was supported as well by the Gordon and Betty Moore Foundation, the Canada Foundation for Innovation, the Ontario Ministry of Research and Innovation, the National Research Council of Canada, the Natural Sciences and Engineering Research Council of Canada, the British Columbia Knowledge Development Fund, the Association of Universities for Research in Astronomy (AURA) and the U.S. National Science Foundation.

\section{References}

1. J. Vernin and F. Roddier, "Experimental determination of twodimensional spatiotemporal power spectra of stellar light scintillation. Evidence for a multilayer structure of the air turbulence in the upper troposphere," J. Opt. Soc. Am. 63, 270273 (1973).
2. A. Fuchs, M. Tallon, and J. Vernin, "Focusing on a turbulent layer: principle of the generalized SCIDAR," Publ. Astron. Soc. Pac. 110, 86-91 (1998).

3. T. Butterley, R. W. Wilson, and M. Sarazin, "Determination of the profile of atmospheric optical turbulence strength from SLODAR data," Mon. Not. R. Astron. Soc. 369, 835-845 (2006).

4. A. Tokovinin, "Measurement of seeing and the atmospheric time constant by differential scintillations," Appl. Opt. 41, 957-964 (2002).

5. A. Tokovinin, V. Kornilov, N. Shatsky, and O. Voziakova, "Restoration of turbulence profile from scintillation indices," Mon. Not. R. Astron. Soc. 343, 891-899 (2003).

6. A. Tokovinin and V. Kornilov, "Accurate seeing measurements with MASS and DIMM," Mon. Not. R. Astron. Soc. 381, 11791189 (2007).

7. M. Thomsen, M. Britton., and A. Pickles, "MASS-DIMM Setup at Palomar," presented at American Astronomical Society Meeting 210, Honolulu, Hawaii, 27-31 May 2007, paper 117.01.

8. M. Britton, "The anisoplanatic point-spread function in adaptive optics," Publ. Astron. Soc. Pac. 118, 885-900, (2006).

9. L. Wang, M. Schöck, G. Chanan, W. Skidmore, R. Blum, E. Bustos, S. Els, R. Riddle, J. Seguel, T. Travouillon, J. Vasquez, D. Walker, and P. Gillett, "High accuracy DIMM measurements for the TMT site testing program," Appl. Opt. 118, 885-900, (2007).

10. V. Kornilov, A. Tokovinin, N. Shatsky, O. Voziakova, S. Potanin, and B. Safonov, "Combined MASS-DIMM instruments for atmospheric turbulence studies," Mon. Not. R. Astron. Soc. 382, 1268-1278 (2007).

11. V. Kornilov, "The verification of the MASS spectral response," 14 September 2006, http://www.ctio.noao.edu/ atokovin/ profiler/archive.html.

12. A. Tokovinin, "Replication of MASS segmentators," 26 December 2004, http://www.ctio.noao.edu/ atokovin/profiler/archive .html.

13. A. Tokovinin, "Calbration of the MASS time constant measurements," 22 June 2006, http://www.ctio.noao.edu/ $\sim$ atokovin/profiler/archive.html. 